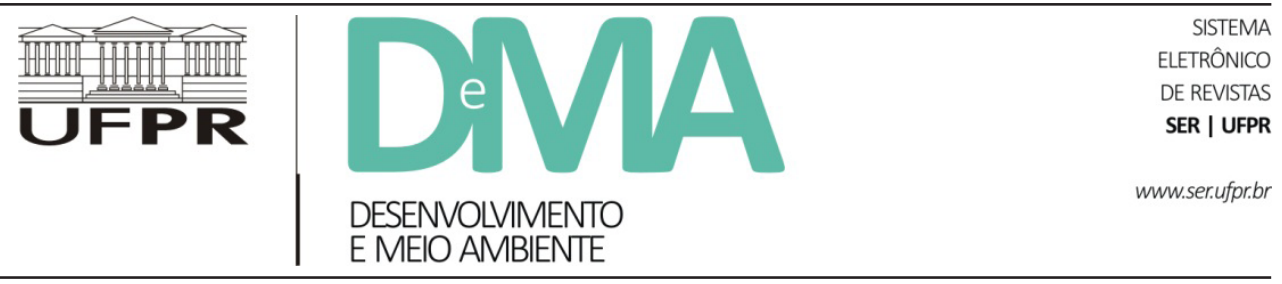

\title{
Análise do nível de intensidade de certificações no padrão ISO 14001: tendências para o continente americano
}

\section{Analysis of the Certification Intensity Levels on ISO 14001: Trends for the American Continent}

\author{
Suzana Eda HIKICHI ${ }^{*}$, Eduardo Gomes SALGADO ${ }^{1}$, Luiz Alberto BEIJO ${ }^{1}$ \\ ${ }^{1}$ Universidade Federal de Alfenas (UNIFAL), Alfenas, MG, Brasil. \\ *E-mail de contato: suzanaeda@hotmail.com
}

Artigo recebido em 27 de janeiro de 2016, versão final aceita em 3 de agosto de 2016.

RESUMO: $\quad$ A busca pela melhoria do desempenho ambiental tem se tornado prioridade em muitas organizações devido à necessidade de demonstrar preocupação para com o meio ambiente. Certificações ambientais, como o padrão ISO 14001, representam estratégias para as empresas aprimorarem sua gestão na área e também como atrativos para consumidores e demais interessados. O número de certificações ISO 14001 emitidas está em constante crescimento em todo o mundo, com mais força em alguns países e menor impulso em outros. Embora a análise do número absoluto de certificados ISO 14001 seja importante, ela não reflete o real interesse das organizações de cada país em obter a certificação. Diante do exposto, o objetivo desta pesquisa foi comparar o número de certificados ISO 14001 nos países do continente americano utilizando um Índice de Intensidade de Certificação, baseado em Casadesús, Marimon e Heras-Saizarbitoria (2008). Esse indicador permite comparar o número de certificações em países com economias de tamanhos diferentes, o que representa diferentes disponibilidades de empresas com viabilidade para certificar. Para isso, foi avaliada a relação entre o número de novas ISO 14001 e o PIB per capita em 13 países do continente americano, nos últimos quatro anos, baseados em dados obtidos junto à ISO (International Organization for Standardization) e o The World Bank. Os resultados mostraram que, enquanto o número de certificados nos Estados Unidos ainda está aquém da sua capacidade, empresas de países como a Colômbia têm demonstrado grande interesse em certificar. Diferentes fatores internos parecem estar motivando ou dificultando a decisão de implantar a ISO 14001 nos países do continente. Compreender esse comportamento é importante para partes as interessadas na ISO 14001, tais como gestores de empresas, comitês técnicos da ISO, governos e órgãos certificadores.

Palavras-chave: ISO 14001; continente americano; intensidade de certificação.

ABSTRACT: The search for improved environmental performance has become a priority in many organizations due to the need showoff showing concern for the environment. Environmental certifications such as ISO 14001 represent strategies for companies to improve their management in the area as well as being attractive to consumers and other interested parties. The number of ISO 14001 certifications issued is constantly growing worldwide, 
stronger in some countries and with lower impulse in others. Although the analysis of the absolute number of ISO 14001 certificates is important, it does not reflect the real interest of the organizations of each country to get certified. Based on the above, the objective of this research was to compare the number of ISO 14001 certificates in the Americas Countries using a Certification Intensity Index, based on Casadesús, Marimon and Heras-Saizarbitoria (2008). This indicator allows the comparison between the number of certifications in countries with economies of different sizes, representing the availabilities of companies with the possibility to get certificated. For this, the relationship between the number of new ISO 14001 and GDP per capita in 13 countries of the Americas in the last four years was evaluated, based on data obtained from ISO (International Organization for Standardization) and The World Bank. The results showed that while the number of certificates in the United States is still below its capacity, companies from countries such as Colombia have shown great interest in getting it. Different internal factors appear to be motivating or complicating the decision to implement ISO 14001 in the continent. Understanding this behavior is important for stakeholders of ISO 14001, such as company managers, ISO technical committees, governments and certification bodies.

Keywords: ISO 14001; Americas; certification intensity.

\section{Introdução}

A crescente preocupação da sociedade com os impactos ambientais dos processos produtivos tem levado a uma valorização da gestão ambiental nas organizações. Interessados em minimizar esses impactos, reduzir custos operacionais e com matéria-prima e, principalmente, melhorar a imagem diante do mercado consumidor, gestores em organizações dos mais diversos setores têm se empenhado em desenvolver políticas e medidas econômicas visando à adoção de práticas mais sustentáveis.

Introduzido em 1996, o sistema ISO 14001 é uma referência internacional utilizada nas organizações para a implantação da gestão ambiental, estrutura utilizada para direcionar a conduta entre a companhia e o meio ambiente (Boiral, 2007; González-Benito et al., 2011). Seguindo o sucesso da norma ISO 9001, o número de companhias que têm adotado o padrão e sendo certificadas vem crescendo a cada ano, com um total de 324.148 certificações emitidas em 170 países ao longo do ano de 2014 (ISO, 2015).

Nos últimos anos, um número significativo de abordagens vem sendo realizado visando entender o processo de crescimento das certificações ao redor do mundo. Porém, a maior parte da literatura na área incide sobre o comportamento das adoções da norma nos países da Europa e da Ásia, raramente sendo estudada a sua disseminação no continente americano.

Entretanto, é perceptível que a ISO 14001 vem adquirindo importância tanto entre as instituições certificadoras como em organizações certificáveis na região, sendo interessante avaliar as perspectivas de evolução na América. Com isso, verifica-se a importância do desenvolvimento de novos trabalhos relacionados ao tema nessa região, caracterizada pela grande desigualdade entre os níveis de desenvolvimento socioeconômico, heterogeneidade cultural e variação no número de certificações ISO 14001 entre os países.

Neste cenário de heterogeneidade socioeconômica dos países do continente americano, e que reflete na quantidade de organizações com interesse e viabilidade de adotar a norma ISO 14001, o objetivo do presente trabalho foi analisar o potencial e o esforço necessário para obtenção da certificação pelas organizações em cada país, além dos números absolutos de certificação. 


\section{Revisão de literatura}

\subsection{O padrão ISO 14001}

A certificação ISO 14001 é o mais reconhecido padrão internacional para o estabelecimento, a implantação, a manutenção ou a melhoria de Sistemas de Gestão Ambiental (SGA). Ele especifica os requisitos para que qualquer empresa ou organização possa configurar um SGA eficaz para desenvolver e implantar "política e objetivos que levem em conta os requisitos legais e outros requisitos que a organização subscreva, e informações sobre aspectos ambientais significativos" (ISO, 2004).

O padrão ISO 14001 foi lançado em setembro de 1996, apresentando estrutura semelhante à norma de Gestão da Qualidade ISO 9001, e sua última revisão foi realizada em 2015. Em relação às versões anteriores, a ISO 14001:2015 busca fortalecer o vínculo entre as estratégias internas da empresa e a proteção ao meio ambiente, além de integrar o conceito de ciclo de vida do produto ou serviço (ISO, 2015c).

O principal objetivo da ISO 14001 é fornecer os requisitos para o estabelecimento de um Sistema de Gestão Ambiental visando melhorar o seu desempenho ambiental e o cumprimento das políticas de conformidade e dos objetivos ambientais (ISO, 2015b). Em nível socioeconômico, a norma visa promover o desenvolvimento sustentável e fomentar o comércio internacional, por meio de um legítimo sistema internacional de normalização que reduza o impacto da diversidade de práticas nacionais, facilitando as trocas comerciais entre países (Bansal \& Hunter, 2003; Casadesús et al., 2008).

De acordo com Potoski \& Prakash (2005), para a obtenção da certificação ISO 14001 as organizações devem adotar um extenso e oneroso SGA. Elas devem realizar uma revisão global de suas práticas e sistemas ambientais, implantar um plano de ação para a gestão ambiental, identificar as responsabilidades internas e planejar a correção dos problemas ambientais existentes.

Woodside et al. (1998) estabelecem que um sistema de SGA em conformidade com a norma ISO 14001 deve seguir o princípio de melhoria contínua, baseado na ferramenta conhecida como ciclo PDCA ou ciclo de Deming (Deming, 1986). Esse ciclo é fundamentado nos seguintes estágios: Plan, Do, Check e Act (Figura 1), e um Sistema de Gestão Ambiental implantado conforme essa metodologia deve apresentar os seguintes requisitos:

1. Uma política ambiental adequada para a organização;

2. Processo de Planejamento para a identificação dos aspectos ambientais e das exigências legais que caracterizam da empresa e, em seguida, implantação de programas ambientais que abordam os impactos considerados significativos;

3. Implantação de um sistema que inclua a estrutura de responsabilidade da empresa para os elementos do SGA: programas de treinamento e conscientização para os funcionários, um sistema de comunicação eficaz, o estabelecimento da documentação do SGA, a identificação de procedimentos de controle operacional, e planos de emergência;

4. Um sistema de verificação e ação corretiva com atividades de monitoramento e medição, análise de não conformidades e ações corretivas, gestão dos registros e agendamento interno de auditorias externas da SGA;

5. Uma revisão da gestão, para verificar e documentar a eficácia e a adequação do SGA com uma frequência estabelecida pela própria empresa. 


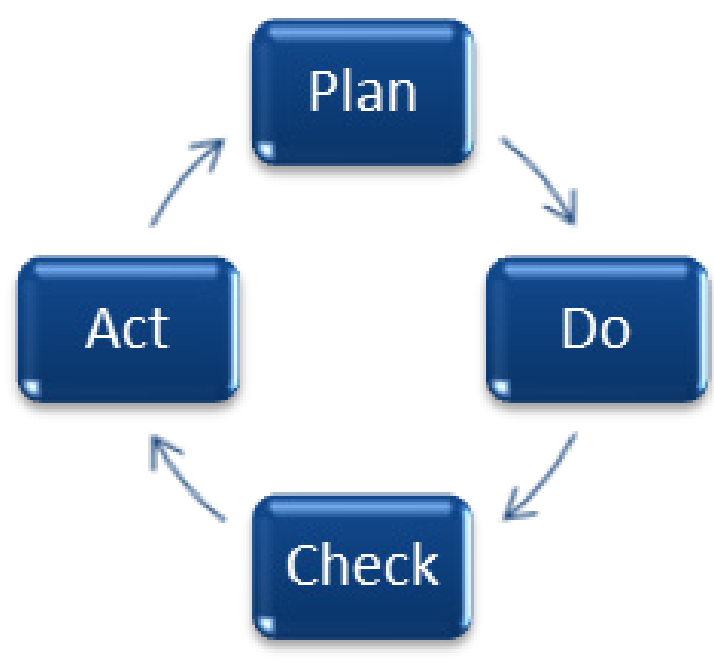

FIGURA 1 - O ciclo PDCA.

FONTE: Autores

A implantação do padrão ISO 14001 assegura que o impacto ambiental das atividades, produtos e serviços dentro da empresa ou organização está sendo mensurado e sistematizado, o que é importante para os gestores e sinaliza compromisso ambiental para com os stakeholders (que são as partes interessadas nas atividades da companhia e incluem funcionários, acionistas, clientes, fornecedores, organizações não governamentais (ONGs), agências governamentais e comunidade local) (Bansal \& Hunter, 2003; ISO, 2009; 2015a).

Na série ISO 14000, a ISO 14001 é o único padrão passível de auditoria para certificação e também o único que fornece uma lista de especificações e requisitos que um SGA precisa cumprir para que a empresa possa ser avaliada e certificada (Neumayer \& Perkins, 2004; González-Benito \& González-Benito, 2005). Assim como outras certificações ISO (como a ISO 9001), a adesão à ISO 14001 é voluntária, ou seja, não há requisitos legais para a certificação, porém, ela geralmente traz inúmeros benefícios tangíveis e intangíveis para as empresas certificadas (Bansal \& Bogner, 2002; Potoski \& Prakash, 2005).

Entre os principais benefícios tangíveis que podem ser proporcionados pela ISO 14001 estão a redução no consumo de recursos e a melhoria na gestão dos resíduos (Bansal \& Bogner, 2002), o maior potencial para conseguir e manter novos negócios (Curkovic \& Sroufe, 2011) e o aumento na produtividade (Barla, 2007), ou seja, benefícios diretamente relacionados a ganhos econômicos. Os possíveis benefícios intangíveis da adoção da certificação ambiental estariam relacionados à melhoria na reputação ambiental da empresa (Potoski \& Prakash, 2005), aos ganhos na relação com os stakeholders e ao aperfeiçoamento do controle organizacional (Poksinska et al., 2003). A ISO 14001 ainda contribui para organizações por ser compatível com os seus princípios de responsabilidade social, qualidade de produtos e processos e com a internacionalização dessas instituições (Bansal \& Hunter, 2003).

Diversos autores relatam benefícios obtidos por empresas após a obtenção da certificação ISO 14001: Zeng et al. (2005) demonstraram a redução da utilização de recursos e geração de resíduos em indústrias selecionadas na China após a implantação da ISO 14001. Em um estudo com empresas sérvias do setor de alimentos, Djekic et al. (2014) verificaram que a adoção da ISO 14001 auxiliou as organizações a alcançarem conformidade legal, melhorou a reputação das empresas e contribuiu na redução da geração de resíduos. Segundo Nguyen \& Hens (2015), melhorias em indicadores ambientais na indústria do cimento do Vietnã também estariam relacionadas com esta certificação.

Entretanto, todo o processo para a implantação da ISO 14001 também envolve custos monetários (tempo, treinamento para operações diárias, manutenção, documentação e preparação para futuras auditorias) e não monetários (descoberta de violações 
regulatórias das empresas nas auditorias externas e exposição pública dessas violações) substanciais para a obtenção da certificação pelas organizações (Delmas, 2002; Kollman \& Prakash, 2002).

Na literatura da ISO 14001 não há consenso sobre quais as situações em que os benefícios da certificação voluntária para as organizações compensam o seu alto custo, principalmente financeiro. Para Nishitani (2010), as firmas adotam a ISO 14001 quando avaliam que os benefícios ultrapassam o alto custo. Segundo Bansal \& Bogner (2002), as empresas investem na implantação da regulação ambiental apenas em circunstâncias específicas como, por exemplo, quando o seu impacto ambiental é grande e também por exigência do mercado internacional. Khan (2008) afirma que os custos são menos restritivos quando os gestores conhecem as vantagens da certificação e o retorno positivo é rápido. Por sua vez, de acordo com Bansal e Hunter, embora gestores não questionem a importância da implantação de um SGA, eles acreditam que a ISO 14001 traz poucos benefícios a um alto custo.

Assim como muitos projetos relacionados ao meio ambiente, o SGA pode não trazer o retorno planejado e os possíveis benefícios institucionais e econômicos podem ser imperceptíveis. Todavia, como argumentam Bansal \& Bogner (2002), um sistema de gestão eficaz gera projetos que podem proporcionar forte ganho financeiro em longo prazo e, além disso, permite que a organização não só atinja seus objetivos e metas ambientais, mas também alcance a melhoria contínua do seu sistema de gestão global.

\subsection{Fatores que motivam a difusão da ISO 14001}

Heras-Saizarbitoria et al. (2011) afirmam que os fatores que levam as organizações a adotarem a ISO 14001, assim como as inovações em geral, po- deriam ser classificados de acordo com a sua origem em pressões externas ou internas. No entanto, na literatura sobre a ISO 14001, ainda não há consenso entre especialistas a respeito de quais seriam as principais motivações para adotar a norma.

Entre as pressões externas para a adoção da ISO 14001 mais observadas na literatura estão a necessidade de demonstrar compromisso ambiental para com stakeholders, pressões da cadeia de suprimentos mundial, redução de barreiras comerciais, competitividade com empresas do mesmo setor, pressões de agências governamentais e organizações não governamentais (ONGs) ligadas ao meio ambiente, distância física dos consumidores e melhoria na imagem corporativa e nas relações com autoridades (Corbett \& Kirsch, 2001; Bansal \& Hunter, 2003; Ghisellini \& Thurston, 2005; Massoud et al., 2010a; Nishitani, 2010).

Por sua vez, as motivações internas para uma empresa adotar a ISO 14001 estão geralmente relacionadas à implantação do SGA para a melhoria do desempenho ambiental e os efeitos dessa mudança na gestão ambiental sobre a estrutura interna da organização. Entre os fatores citados na literatura estão a busca por melhoria na eficiência e na eficácia nas operações internas da companhia, redução dos impactos ambientais dos processos de produção, com simultânea queda na geração de resíduos e economia nos custos, além de questões éticas (Bansal \& Bogner, 2002; Massoud et al., 2010a; Kola-Lawal et al., 2014).

A norma ISO 9001, que estabelece os requisitos para o estabelecimento de um Sistema de Gestão da Qualidade, é reconhecida como uma grande influência na decisão de adotar a ISO 14001, com organizações mais propensas a adotar a ISO 14001 em países em que a certificação da qualidade é mais presente (Vastag, 2004). Em um estudo com 56 países, Albuquerque et al. (2007) verificaram 
que, em razão das semelhanças na concepção, na linguagem, na estrutura e na metodologia entre os padrões, a adoção prévia da norma ISO 9001 facilitaria a implantação e contribuiria para uma difusão mais rápida da ISO 14001. Ainda segundo Albuquerque et al. (2007, p. 454), esse fenômeno ocorreria pelo fato de a disseminação da ISO 9001 "reduzir as incertezas relacionadas ao valor comercial e à relevância dos padrões de normalização". As semelhanças entre as motivações para adotar as certificações ISO 14001 e ISO 9001 também foram destacadas por Corbett \& Kirsch $(2001 ; 2004)$, Vastag (2004) e Viadiu et al. (2006).

Também segundo Albuquerque et al. (2007), entretanto, há diferenças nos fatores que direcionam a difusão das duas normas entre os países do mundo: embora a difusão de ambas, ISO 9001 e ISO 14001, seja guiada pela proximidade geográfica entre os países, a ISO 9001 seria influenciada principalmente por fatores econômicos, enquanto as razões para adotar a ISO 14001 estariam mais relacionadas à similaridade cultural entre os países.

Corbett \& Kirsch (2001), em um estudo com 63 países, observaram que, além da influência ambiental, a força de exportação e o Produto Interno Bruto do país seriam motivações significativas para a difusão da ISO 14001. Utilizando o mesmo grupo de dados, Vastag (2004) também verificou que os fatores econômicos seriam importantes para a difusão da ISO 14001, pois o foco do país em exportações, dado pela porcentagem de exportações no total do Produto Interno Bruto nacional, pode forçar empresas a adotar a ISO 14001 se o padrão for exigido no mercado de destino. Da mesma forma, em uma análise mundial, To e Lee (2014) constataram que há uma correlação positiva entre a adoção da ISO 14001 e a intensidade do comércio internacional, principalmente nos primeiros estágios da difusão mundial da norma.

\subsection{O Índice de Intensidade de Certificação}

Para uma análise mais detalhada da evolução da ISO 14001 em nível de país, paralelamente à análise dos números absolutos da certificação, é interessante dimensionar o número de certificados emitidos considerando o potencial econômico do país, com o objetivo de melhor confrontar nações com economias com diferentes capacidades (Casadesus et al., 2008; Marimon et al., 2010).

Com essa finalidade, um Índice de Intensidade de Certificação para a ISO 14001 e a ISO 9001 foi inicialmente proposto por Marimon et al. (2006), visando medir a porcentagem de certificações em cada um dos países da Europa selecionados para o estudo em relação ao total de certificações na Europa e a porcentagem de contribuição de cada um desses países para o PIB total do continente europeu.

Baseados nesse indicador, Casadesús et al. (2008) propuseram uma modificação no índice, comparando os 13 países com os maiores números de certificações ISO 14001 e ISO 9001 usando a relação entre o número de certificados ISO 14001 ou ISO 9001 no país e o Produto Interno Bruto (PIB) do país (1).

$i 14=\frac{\text { Número de certificados ISO } 14001 \text { emitidos no país no ano }}{\text { Média do PIB do país nos últimos quatro anos }}$

em que $i 14=$ Intensidade de Certificações ISO 14001 em determinado ano.

Entretanto, devido às flutuações nos preços e nas moedas de cada país, o que dificulta comparações internacionais utilizando-se o PIB, Casadesús, Marimon e Heras-Saizarbitoria (2008) sugeriram também que a melhor alternativa seria utilizar o número de companhias ou de plantas industriais no 
país ao invés do PIB, o que é, no entanto, segundo os autores, inviabilizado pela dificuldade de acesso a esses dados. Ainda com relação a essa flutuação nos preços e moedas, Marimon et al. (2010), em um estudo com as nações líderes no número de certificações ISO 14001 e ISO 9001, propuseram um novo indicador de Intensidade de Certificações, substituindo o PIB do país pelo Produto Nacional Bruto medido em Paridade do Poder de Compra (PNB/PPC), que é calculado considerando-se o rendimento nacional bruto (convertido para dólares internacionais) em termos de paridade de poder aquisitivo.

\section{Metodologia}

Em virtude da natureza dos dados, o desenho de pesquisa aplicado no neste artigo foi o cross-national. O presente trabalho foi desenvolvido por meio de análise exploratória do número de certificações ISO 14001 emitidas para os países do continente americano entre os anos de 1996 e 2014, medição da Intensidade de Certificação ISO 14001 nos países do continente, utilizando uma versão modificada do indicador proposto por Casadesús et al. (2008), e revisão bibliográfica sobre a difusão das certificações ISO 14001 nos países do continente.

\subsection{Dados}

Os dados de ISO 14001 utilizados na execução do trabalho foram obtidos em ISO (2000) e ISO (2015). Foi avaliado o número de certificações emitidas para países do continente americano entre os anos de 1996 e 2014.

$\mathrm{Na}$ avaliação da Intensidade de Certificação da ISO 14001 nas Américas, foram selecionados os 13 países do continente que apresentaram número de certificações ISO 14001 maior que 50 no ano de 2014 (Argentina, Bolívia, Brasil, Canadá, Chile, Colômbia, Costa Rica, Equador, Estados Unidos, México, Peru, Uruguai e Venezuela).

Este grupo de países respondeu por quase $99 \%$ do total de certificados emitidos no continente americano naquele ano.

A escolha destes 13 países se justificou pelo fato de que países que apresentaram desempenho abaixo de 50 certificados foram considerados não representativos para a análise da difusão dessa certificação no continente. Além disso, a natureza da análise quantitativa e descritiva torna difícil trabalhar com uma grande quantidade de países, sendo selecionados apenas aqueles com maior relevância para o continente.

\subsection{Avaliação da Intensidade da Certificação ISO 14001 na América}

A análise em nível de país no continente foi realizada por meio da aplicação de Índice de Intensidade de Certificação da ISO 14001 (i14) baseado no indicador desenvolvido por Casadesús et al. (2008). Nesse trabalho, propôs-se avaliar a relação do número de certificados ISO $14001 \mathrm{com}$ o PIB per capita (PIBpc), que consiste no Produto Interno Bruto dividido pelo número de habitantes do país (The World Bank, 2015). Esse indicador é amplamente utilizado internacionalmente para a comparação do padrão de renda entre países, apesar de não incorporar a desigualdade de renda.

Para isso, foram propostas uma modificação e uma nova aplicação para o indicador Índice de Intensidade de Certificação em ISO 14001, criado por Casadesús et al. (2008), visando comparar o número de certificados existentes em países do continente americano, entre os anos de 2010 e 2014, tendo em conta as disparidades econômicas entre 
estes países. A escolha do espaço temporal dos cinco últimos anos foi considerada adequada por abranger o momento de difusão da certificação ISO 14001 na maioria dos países do continente americano.

Com essa modificação no Índice, a proposta foi melhorar o dimensionamento do número de certificados ISO 14001 emitidos para os países, tendo em conta o potencial econômico nacional, com relação aos resultados obtidos em estudos anteriores (Casadesús et al., 2008; Marimon et al., 2010). A utilização do PIBpc em substituição ao PIB total possibilita comparar padrões de renda entre países e reduz problemas relacionados ao PIB total, como as flutuações em preços e moedas, sendo apropriada para comparar o potencial e o esforço dos países para a obtenção da certificação ISO 14001.

O Índice de Intensidade de Certificação na ISO 14001 (i14) foi calculado usando a razão entre o número de certificados ISO 14001 emitidos no país em um ano e o PIB per capita (PIBpc) do país naquele ano (2),

$i 14=\frac{\text { Número de certificados ISO } 14001 \text { emitidos no país }}{\text { PIB per capita do país }}$
Baseada no i14 obtido para cada país, foi realizada uma análise dos fatores que vêm motivando ou dificultando a difusão da ISO 14001 entre os países.

\section{Resultados e discussões}

\subsection{Evolução no número de certificações ISO 14001 do continente americano}

O número de certificações ISO 14001 no continente americano está em constante crescimento nos últimos anos, apesar da queda no volume de certificados emitidos entre os anos de 2007 e 2009 (Figura 2). No final de 1996, ano em que a ISO 14001 foi lançada, o total de certificados na região era de apenas 54 (ISO, 2000). No ano de 2006, dez anos após a divulgação da norma, o número de certificados emitidos foi de 12.028 e em 2014 esse número quase dobrou, sendo concedidas 20.282 certificações no total (ISO, 2015). O comportamento da evolução da ISO 14001 nos países americanos está apresentado na Figura 2.

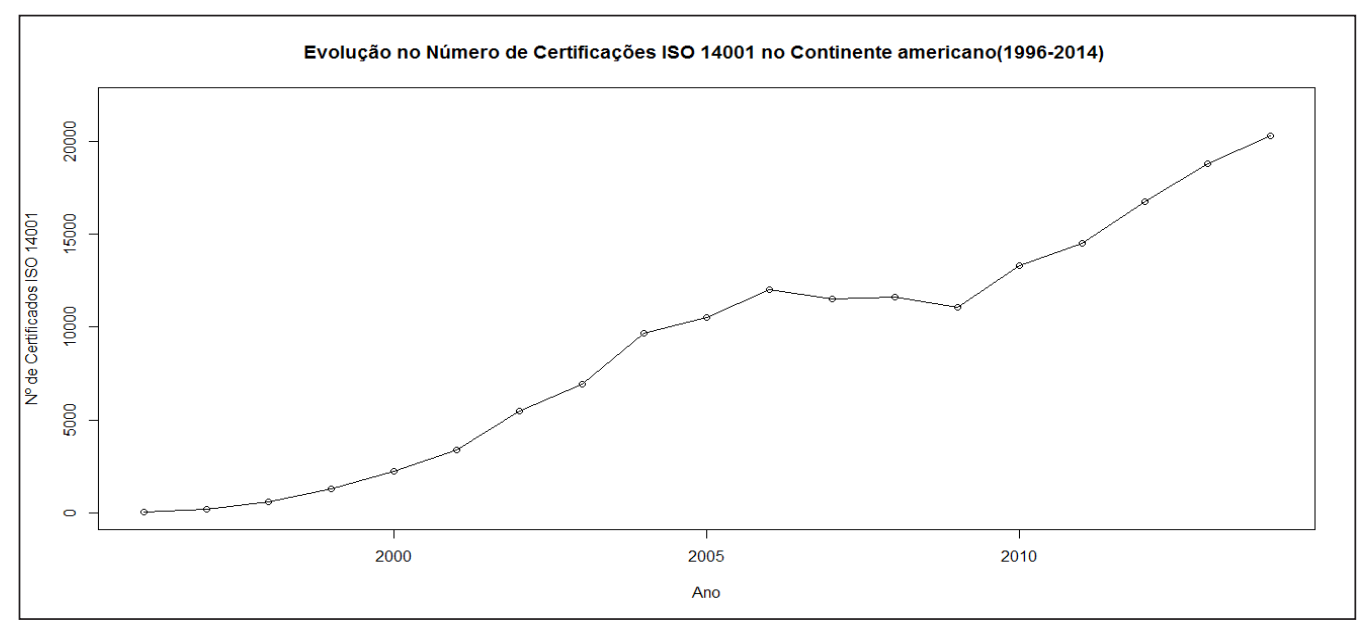

FIGURA 2 - Evolução no número de certificações ISO 14001 emitidas no continente americano entre os anos de 1996 e 2014. FONTE: ISO $(2000 ; 2015)$. 
A ISO 14001 foi implantada por organizações na maior parte dos países do continente americano, estando presente nos três subcontinentes, América do Norte, América Central e América do Sul. Estados Unidos, Colômbia e Brasil se sobressaem como as nações onde as organizações obtiveram o maior número de certificações nos últimos anos (Tabela 1), representando em conjunto mais de $60 \%$ do total emitido em todo o continente no ano de 2014 (ISO, 2015).

TABELA 1 - Os 13 países com maior número de certificações ISO 14001 no continente americano em 2014.

\begin{tabular}{ccc}
\hline Posição & País & Número de certificados \\
\hline 1 & Estados Unidos & 6.586 \\
2 & Colômbia & 3.453 \\
3 & Brasil & 3.222 \\
4 & Canadá & 2.101 \\
5 & México & 1.452 \\
8 & Peru & 353 \\
9 & Equador & 189 \\
6 & Argentina & 1.341 \\
7 & Chile & 967 \\
10 & Uruguai & 147 \\
11 & Costa Rica & 91 \\
12 & Venezuela & 76 \\
13 & Bolívia & 52 \\
\hline
\end{tabular}

FONTE: ISO (2015).

\subsection{Intensidade de Certificações ISO 14001 no continente americano}

Com o objetivo de comparar o número de certificados ISO 14001 emitidos nos países do continente americano, os valores foram dimensionados por meio da relação entre o número de certificações ISO 14001 do país no ano e o PIBpc nacional do mesmo ano, gerando o valor de $i 14$. Os resultados estão apresentados na Tabela 2.
TABELA 2 - Número de certificações ISO 14001/PIBpc* por ano para os países do continente americano (2010-2014).

\begin{tabular}{cccccc}
\hline País & $\mathbf{2 0 1 0}$ & $\mathbf{2 0 1 1}$ & $\mathbf{2 0 1 2}$ & $\mathbf{2 0 1 3}$ & $\mathbf{2 0 1 4}$ \\
\hline Argentina & 76,595 & 58,334 & 87,832 & 89,445 & 106,695 \\
Bolívia & 11,609 & 19,358 & 16,664 & 18,012 & 16,070 \\
Brasil & 304,834 & 269,663 & 276,783 & 315,514 & 283,013 \\
Canadá & 22,902 & 31,390 & 33,717 & 33,935 & 41,793 \\
Chile & 55,846 & 42,312 & 70,804 & 62,700 & 66,560 \\
Colômbia & 166,223 & 182,214 & 182,751 & 347,036 & 436,872 \\
Costa Rica & 11,896 & 8,144 & 8,322 & 7,647 & 8,737 \\
Equador & 30,060 & 21,634 & 26,572 & 33,321 & 29,894 \\
Estados & 91,103 & 99,575 & 110,753 & 114,590 & 120,558 \\
Unidos & 91,181 & 88,178 & 112,745 & 104,992 & 141,933 \\
México & 9,307 & 43,271 & 46,175 & 51,959 & 53,886 \\
Peru & 79,307 & 7,820 & 8,746 \\
Uruguai & 9,047 & 7,977 & 7,734 & 7,930 & 4,574 \\
Venezuela & 4,860 & 4,556 & 7,282 & 6,930 \\
\hline FONTE: Elaborado de informações ISO (2015) e The World Bank \\
(2015). \\
* PIBpc expresso por 1mil habitantes.
\end{tabular}

Apesar de os índices da Tabela 2 não permitirem inferir sobre a evolução dessa intensidade de certificações ISO 14001 ao longo dos anos dentro de cada país, por depender de duas variáveis, o PIB e o número de habitantes, fatores que, assim como a ISO 14001, variam ao longo do tempo, eles possibilitam comparar o empenho das empresas de diferentes países em buscar a certificação dentro de um mesmo ano.

Na comparação entre países, a Colômbia é o país cujas organizações vêm demonstrando o maior interesse em certificar desde 2013, embora os Estados Unidos apresentem maiores números absolutos de certificações. Observa-se também que os Estados Unidos, embora possuam o maior número de certificados entre os países do continente, apresentavam em 2014 um $i 14$ menor do que países como Colômbia, Brasil e México, o que mostra que o número de certificados ISO 14001 pode não refletir um baixo empenho do país em adotar a norma de gestão ambiental, em relação ao tamanho da sua economia. Na América Latina, além da Colômbia, 
destacam-se Brasil, México, Argentina. Os três países estão entre as nações com o maior número de certificados ISO 9001 obtidos nos últimos anos (ISO, 2015), o que mostra que esse pode também ser um fator positivo para a difusão do número de certificações ISO 14001 nesses países.

No continente americano, os Estados Unidos apresentam um comportamento particular. Eles são o país com a maior economia mundial, com um Produto Interno Bruto (PIB) que correspondia a aproximadamente $22 \%$ do total do PIB mundial em 2014, lideram o comércio mundial de mercadorias, ao lado da China, e estavam entre os países com o maior Índice de Desenvolvimento Humano (IDH), ocupando a $5^{\mathrm{a}}$ posição em 2013 (Malik et al., 2014; World Trend Organization, 2014; The World Bank, 2015). Entretanto, os EUA não apresentam o maior índice $i 14$ no continente (Tabela 2). O número absoluto de certificados é baixo em comparação a países desenvolvidos europeus e da Ásia, como o Japão, e muitas das decisões de certificar dentro dos Estados Unidos foram feitas por companhias estrangeiras (Bansal \& Bogner, 2002; Delmas, 2002).

O ambiente cultural institucional desfavorável ao desenvolvimento da ISO 14001 nos Estados Unidos é apontado por Delmas (2002) como um dos principais fatores limitantes do crescimento no número de certificações no país. Segundo Delmas (2002, p. 106), ao contrário dos países europeus, o ambiente institucional dos Estados Unidos é caracterizado por um "um comando muito sofisticado e um sistema de controle de regulações em que a ISO 14001 teria dificuldade para encontrar espaço". Além disso, a falta de experiência anterior nos procedimentos para implantação de SGA, de informações sobre a ISO 14001 e de apoio das autoridades também estariam desfavorecendo o crescimento nas adoções do padrão ISO 14001 no país (Kollman \& Prakash, 2002).

Ao contrário de outros países desenvolvidos, principalmente da Europa, onde a relação amistosa entre órgãos reguladores e companhias beneficia o alto número de adoções ISO 14001, as companhias nos Estados Unidos não estariam interessadas em implantar a norma por temer a exposição de seu desempenho ambiental ao público e a descoberta de violações regulatórias durante o processo de certificação do SGA, devido à falta de confiança nos órgãos auditores e à adversidade entre indústrias e agências regulatórias do governo no país (Delmas, 2002; Jiang \& Bansal, 2003). Ainda neste país, a Agência de Proteção Ambiental dos Estados Unidos - Environmental Protection Agency (EPA), agência governamental responsável por redigir regulamentações necessárias para a implantação de leis, suportar e promover a ISO 14001, opõe-se à proteção da auditoria, desmotivando empresas com problemas judiciais ambientais a certificarem suas estruturas (Environmental Protection Agency, 2012; Potoski \& Prakash, 2005).

Bansal e Bogner (2002), Delmas (2002) e Kollman e Prakash (2002) também comentaram sobre a influência cultural no baixo interesse em obter a ISO 14001 nos Estados Unidos. Segundo os autores, embora os gestores acreditem nos efeitos positivos da certificação ISO 14001 no desempenho ambiental, eles apresentam pouco interesse em certificar as suas companhias pelo fato de o padrão não ser exigido pelo mercado, pela fraca relação das organizações com stakeholders e para com os próprios competidores organizacionais e também pelo próprio baixo número de companhias certificadas.

Os fatores apresentados para explicar o reduzido empenho das organizações em certificar nos Estados Unidos também podem ser aplicados para 
o Canadá, em vista da proximidade geográfica, similaridade cultural e também porque são os parceiros comerciais mais fortes um do outro (Bansal \& Bogner, 2002; Albuquerque et al., 2007; World Trade Organization, 2014). Para aumentar o número de certificações, Jiang \& Bansal (2003) sugeriram, em um estudo empírico com companhias canadenses do setor de papel e celulose, que os governos aumentem a pressão sobre a cadeia de suprimentos, para que empresas grandes e influentes no mercado exijam a certificação de seus fornecedores.

O México apresenta boa intensidade de certificação i14 (Tabela 2) em relação ao número absoluto de certificados ISO 14001. O meio ambiente tem se tornado uma questão prioritária para o governo mexicano nos últimos anos, principalmente após a adesão ao Tratado Norte-Americano de Livre Comércio - North American Free Trade Agreement - (NAFTA) e posteriormente, à Organização para a Cooperação e Desenvolvimento Econômico - Organization for Economic Co-operation and Development (OECD), fato que está motivando organizações públicas e privadas a aderirem a iniciativas de gestão ambiental (Montiel \& Husted, 2009).

Segundo Montiel \& Husted (2009), as empresas no México têm a opção de escolher entre dois programas de gestão: a norma ISO 14001 e a regulação nacional Certificação de Indústria Limpa - Certificación de Industria Limpia -, sendo que organizações mexicanas envolvidas em negócios com o governo tendem a adotar a certificação nacional, enquanto as multinacionais que operam no país geralmente são inclinadas a implantar a ISO 14001. A norma ISO 14001 sofre competição com a regulação ambiental nacional, que muitas vezes é vantajosa para empresas que visam apenas atender a legislação ambiental nacional, o que pode vir a comprometer o número de certificações ISO 14001 , apesar do bom empenho ambiental do país.
As regulações obrigatórias nacionais, cujo descumprimento é punido por meio de multas, funcionam como uma das motivações para as empresas mexicanas certificarem, atraindo companhias "sujas" sobre pressões de agências regulatórias (Blackman, 2012). O México se caracteriza pela alta especificidade do perfil das empresas mais propensas a certificar: companhias exportadoras, que utilizam matéria-prima importada, relativamente grandes (medida por receita bruta ou capitalização) e pertencentes a setores e estados geográficos característicos (Blackman \& Guerrero, 2012). Segundo Blackman (2012), a maioria das certificações ISO 14001 foi emitida especificamente para instalações estatais de geração de energia elétrica (de propriedade da Comisión Federal de Electricidad) e refinarias de petróleo e terminais de distribuição (de propriedade da Petróleos Mexicanos), que mantém operações no exterior.

No Brasil, a nova estratégia de desenvolvimento socioeconômico que surgiu na década de 1990, representada pela liberalização do comércio (que levou a uma mudança no foco do mercado) e pelos incentivos para o aumento da importação e exportação (com consequente exposição das companhias brasileiras à competição internacional), além da mudança na consciência ambiental da sociedade, foram fatores fundamentais para a consolidação da ISO 14001 no país, que apresentava o terceiro maior número de certificados ISO 14001, entre os países do continente americano, em 2014 (Abreu, 2009; ISO, 2015).

Apesar do grande esforço de empresas brasileiras para obter a ISO 14001 (Tabela 2), o número de certificados emitidos ainda é limitado pelo perfil das companhias nacionais, predominado por pequenas e médias empresas (Ambrosini et al., 2014), em que as dificuldades para implantação desta estratégia ambiental são ainda maiores (Dahlmann et al., 2007). 
Entretanto, segundo Baumbach et al. (2013), as perspectivas para o crescimento da ISO 14001 no Brasil são boas, e estes autores relatam o interesse cada vez maior na implantação da ISO 14001 pelas pequenas e médias empresas, que formam a maioria das companhias operando no Brasil e constituem de 80-90\% do total de empresas da América Latina.

Analisando o panorama das certificações no Brasil, os maiores responsáveis pelo bom desempenho da norma foram os estados da região Sudeste, principalmente São Paulo e Rio de Janeiro, dois grandes parques industriais do país (Pombo \& Magrini, 2008). Esta é a região brasileira que concentra o maior número de empresas de médio ou grande porte, que representam a maioria das empresas certificadas pela norma ISO 14001 (Baumbach et al., 2013).

No Chile, as organizações receberam apenas 967 certificações ISO 14001 no ano de 2013 (ISO, 2014). Entretanto, o país apresentava um bom valor de $i 14$ (Tabela 2), principalmente quando avaliado o tamanho da sua economia. A intensificação da postura ambiental ativa, principalmente após o retorno à democracia na década de 1990, e a participação do setor minerador podem justificar o interesse chileno na implantação da norma ISO 14001.

A indústria mineradora teve grande importância para o crescimento no número de certificações ISO 14001 no país. Ela constituía o principal setor industrial da economia chilena, formado em sua maioria por empresas de grande porte, sendo responsável por aproximadamente $40 \%$ das exportações (Newbold, 2006). Ainda segundo Newbold (2006), as companhias do setor de mineração, considerado relativamente "sujo" e perigoso, se interessavam em implantar SGAs certificados com a ISO 14001 para demonstrar comprometimento com os stakeholders e favorecer futuras negociações. Visto que a grande maioria das principais mineradoras foi certificada, esse fato contribuiu fortemente para a disseminação da norma em nível nacional, impulsionando fornecedores e outras companhias a também implantarem práticas de gestão ambiental.

A aplicação de abordagens voluntárias (AV), uma forma de regulação informal, pelos governos, pode estar estimulando o crescimento no número de adoções de práticas ambientais como a ISO 14001. Governos de países com bons valores de i14 (Tabela 2), como Brasil, Chile, Colômbia e México, têm aplicado as AV por meio de programas de divulgação de informações, subsídios para o controle de poluição e alívio regulatório para participação nos programas de certificação (Earnhart et al., 2014). As abordagens visam promover a melhoria no desempenho ambiental das organizações, buscando reduzir os custos com a prevenção e o controle da poluição e podem aumentar a probabilidade de que essas companhias certifiquem com a ISO 14001 (Wu et al., 2007; Blackman, 2010).

De forma geral, as motivações que levam empresas, em países com vários graus de desenvolvimento socioeconômico, a certificarem, estão relacionadas a pressões externas de consumidores, à competitividade industrial, à melhoria da imagem corporativa, ao acesso a outros mercados e à melhoria interna no desempenho ambiental para redução de custos. No entanto, embora compartilhem essas características, as razões que levam empresas em países industrializados e em desenvolvimento a obter certificações ambientais voluntárias também se diferenciam, pois o contexto socioeconômico e os objetivos das companhias nesses países são distintos (Blackman, 2008; 2012). Enquanto em países desenvolvidos as regulações ambientais são aplicadas para incentivar organizações a tentar ir além da conformidade, empresas em países em desenvolvimento visam, como afirma Blackman (2008), "sanar o descumprimento desenfreado da regulamentação obrigatória”. 
Nos países da América Latina, a abertura política em relação ao comércio e ao capital internacional, em meados da década de 1990, levou a um cenário favorável que propiciou boas taxas crescimento no número de certificações ISO 14001 (Bodas-Freitas \& Izuka, 2008). De forma complementar, Bodas-Freitas \& Izuka (2008) sugerem que essa difusão dos certificados ISO 14001 na região estaria relacionada à infraestrutura e ao nível de desenvolvimento econômico, à capacidade de exportar produtos com relativa sofisticação tecnológica e à velocidade de crescimento do Investimento Direto Estrangeiro (IDE).

O número de certificações emitidas para organizações nos países em desenvolvimento da América Latina, assim como para nações em desenvolvimento em geral, contudo, ainda é relativamente baixo. Esse fenômeno pode ser explicado pelas muitas dúvidas existentes a respeito da eficácia da regulação ambiental voluntária nesses países, o que reduziria a importância da implantação de SGA certificados. As pressões regulatórias e não regulatórias fracas, a captura regulatória e o predomínio de companhias pequenas e informais são argumentos citados por Blackman (2008) como preocupações a respeito da difusão da regulação voluntária nos países em desenvolvimento.

Principalmente na América Central, quando há regulação ambiental, muitas vezes ela é ineficaz devido à escassez de recursos financeiros e de pessoal treinado, à infraestrutura, às condições precárias das instituições responsáveis por aplicar a legislação e à mesmo falta de vontade política, o que dificulta a emissão de novas certificações (Mauri, 2002; Fikru, 2014). O fato de a maioria das empresas latino-americanas serem de pequeno e médio portes faz com que as certificações ambientais se disseminem com maior dificuldade, pois o custo para a certificação, geralmente alto, se torna inviável para essas companhias (Massoud et al., 2010b). A composição da economia, principalmente voltada para a agricultura, que, apesar do foco em exportação, não possui muitas certificações em comparação ao setor industrial, pode também ter efeito negativo nas certificações da região (ISO, 2014; World Trade Organization, 2014).

Especificamente em relação aos países do continente americano que apresentaram número de certificações menores que 50 certificados nos anos de 2013/2014, a baixa adesão parece estar relacionada às características intrínsecas da economia e ao reduzido potencial econômico e, por consequência, o número limitado de companhias com interesse e viabilidade econômica para implantar a norma ISO 14001.

\section{Considerações finais}

No continente americano, a norma de gestão ambiental ISO 14001 está presente na grande maioria dos países e o número de novas certificações está em constante crescimento nos últimos anos, apesar da disparidade socioeconômica entre os países e das instabilidades relacionadas principalmente ao cenário econômico no continente.

O Índice de Intensidade de Certificação da ISO 14001, utilizado para demonstrar o interesse que cada país está apresentando em obter a certificação ISO 14001, revelou que, embora os Estados Unidos sejam o país com o maior número de certificações no continente, suas organizações não seriam aquelas mais empenhadas em implantar a norma ambiental, apesar de possuírem a viabilidade, principalmente financeira, necessária para certificarem suas estruturas. Em relação ao tamanho da sua economia, a Colômbia, que já se destaca em relação ao número absoluto de certificados ISO 14001, tem mostrado, de acordo com o índice, que suas empresas têm 
apresentado grande interesse em implantar Sistemas de Gestão Ambiental para obtenção da certificação.

Os fatores que estão motivando ou dificultando a decisão de implantar a ISO 14001 parecem se diferenciar entre os países do continente. $\mathrm{O}$ ambiente cultural institucional desfavorável e a fraca pressão comercial seriam alguns dos principais fatores que desmotivariam empresas norte-americanas a buscar a certificação. Nos países latino-americanos, embora a abertura político-econômica nas últimas décadas tenha propiciado um cenário favorável, a implantação de regulações ambientais voluntárias ainda é dificultada por deficiências na infraestrutura e pelos altos custos do processo de certificação. $O$ crescimento da preocupação ambiental por consumidores, no entanto, vem gerando pressões para a adoção de certificações visando à melhoria da imagem corporativa.

O conhecimento e as ideias abordadas podem ser de interesse acadêmico e aplicadas por gestores empresariais e organismos certificadores. Os resultados do presente estudo requerem validação em estudos subsequentes e podem servir como base para trabalhos que visem aprofundar essa discussão e estudar as perspectivas futuras sobre a difusão da ISO 14001 no continente americano. Como sugestões de trabalhos futuros estão: uma análise comparativa dos resultados das diferentes versões do Índice de Intensidade de Certificação; a análise dessa Intensidade de Certificação em diferentes setores econômicos; e a modelagem estatística desses dados ISO 14001 nos países do continente americano.

\section{Agradecimentos}

Os autores gostariam de agradecer à UNIFAL-MG e à CAPES pelo apoio por meio da cessão das bolsas de Mestrado.

\section{Referências}

Abreu, M.C.S. de. How to define an environmental policy to improve corporate sustainability in developing countries. Business Strategy and the Environment, 18(8), 542-556, 2009. doi: $10.1002 /$ bse. 625

Albuquerque, P.; Bronnenberg, B. J.; Corbett, C. J. A spatiotemporal analysis of the global diffusion of ISO 9000 and ISO 14000 certification. Management Science, 53(3), 451-468, 2007. doi: 10.1287/mnsc.1060.0633

Ambrosini et al. As micro e pequenas empresas na exportação brasileira: 1998-2013. 2014. Disponível em: <http://www. sebrae.com.br/Sebrae/Portal\%20Sebrae/Anexos/As\%20 MPE\%20na\%20expota\%C3\%A7\%C3\%A3o\%20brasileira_estados_1998\%202013.pdf>. Acesso em: fev. 2015.

Bansal, P.; Bogner, W. C. Deciding on ISO 14001: economics, institutions, and context. Long Range Planning, 35(3), 269-290, 2002. doi: 10.1016/S0024-6301(02)00046-8
Bansal, P.; Hunter, T. Strategic explanations for the early adoption of ISO 14001. Journal of Business Ethics, 46(3), 289-299, 2003. doi: 10.1023/A:1025536731830

Barla, P. ISO 14001 certification and environmental performance in Quebec's pulp and paper industry. Journal of Environmental Economics and Management, 53(3), 291306, 2007. doi: 10.1016/j.jeem.2006.10.004

Baumbach, M. de O.; Prado Filho, J. F. do; Fonseca, A. Environmental management in small mining enterprises: comparative analysis of three Brazilian cases through the lenses of ISO 14001. REM: Revista Escola de Minas, 66(1), 111-116, 2013. doi: 10.1590/S0370-44672013000100015

Blackman, A. Can voluntary environmental regulation work in developing countries? Lessons from case studies. Policy Studies Journal, 36(1), 119-141, 2008. doi: 10.1111/j.15410072.2007.00256.x 
Blackman, A. Alternative Pollution Control Policies in Developing Countries. Review of Environmental Economics and Policy, 4(2), 234-253, 2010. doi: 10.1093/reep/req005

Blackman, A. Does eco-certification boost regulatory compliance in developing countries? ISO 14001 in Mexico. Journal of Regulatory Economics, 42(3), 242-263, 2012. doi: 10.1007/s11149-012-9199-y

Blackman, A.; Guerrero, S. What drives voluntary ecocertification in Mexico? Journal of Comparative Economics, 40(2), 256-268, 2012. doi: 10.1016/j.jce.2011.08.001

Bodas-Freitas, I. M.; Izuka, M. Standards compliance as an alternative learning opportunity under globalization in Latin America. In: Spru-Science and Technology Policy Research. Brighton, 2008. p. 0-37.

Boiral, O. Corporate Greening Through ISO 14001: A Rational Myth? Organization Science, 18(1), 127-146, 2007. doi: 10.1287/orsc. 1060.0224

Casadesus, M.; Marimon, F.; Heras-Saizarbitoria, I. ISO 14001 diffusion after the success of the ISO 9001 model. Journal of Cleaner Production, 16(16), 1741-1754, 2008. doi: 10.1016/j.jclepro.2007.11.002

Corbett, C. J.; Kirsch, D. A. International diffusion of ISO 14000 certification. Production and Operations Management, 10(3), 327-342, 2001. doi: 10.1111/j.1937-5956.2001. tb00378.x

Corbett, C. J.; Kirsch, D. A. Response to "Revisiting ISO 14000 Diffusion: A New Look at the Drivers of Certification". Production and Operations Management, 13(3), 268-271, 2004. doi: 10.1111/j.1937-5956.2004.tb00511.x

Curkovic, S.; Sroufe, R. Using ISO 14001 to promote a sustainable supply chain strategy. Business Strategy and the Environment, 20(2), 71-93, 2011. doi: 10.1002/bse.671

Dahlmann, F.; Brammer, S.; Millington, A. Environmental management in the United Kingdom: new survey evidence. Management Decision, 46(2), p. 264-283, 2007. doi: $10.1108 / 00251740810854159$

Delmas, M. A. The diffusion of environmental management standards in Europe and in the United States: An institutional perspective. Policy Sciences, 35(1), 91-119, 2002. doi: 10.1023/A:1016108804453

Deming, W. E. Out of the crisis. Massachusetts Institute of Technology. Center for Advanced Engineering Study, Cambridge, MA, 1986.
Djekic, I. et al. Environmental management effects in certified Serbian food companies. Journal of Cleaner Production, 76, 196-199, 2014. doi: 10.1016/j.jclepro.2014.04.062

Earnhart, D. H.; Khanna, M.; Lyon, T. P. Corporate environmental strategies in emerging economies. Review of Environmental Economics and Policy, 8(2), 164-185, 2014. doi: 10.1093/reep/reu001

EPA - Environmental Protection Agency. Voluntary Environmental Management System/ISO 14001. 2012. Disponível em: $<$ http://water.epa.gov/polwaste/wastewater/ Voluntary-EMS-ISO-14001.cfm>. Acesso em: set. 2015.

Fikru, M. G. International certification in developing countries: The role of internal and external institutional pressure. Journal of Environmental Management, 144, 286-296, 2014. doi: 10.1016/j.jenvman.2014.05.030

Ghisellini, A.; Thurston, D. L. Decision traps in ISO 14001 implementation process: case study results from Illinois certified companies. Journal of Cleaner Production, 13(8), 763-777, 2005. doi: 10.1016/j.jclepro.2004.02.042

González-Benito, J.; González-Benito, O. An analysis of the relationship between environmental motivations and ISO14001 certification. British Journal of Management, 16(2), 133-148, 2005. doi: 10.1111/j.14678551.2005.00436.x

González-Benito, J.; Lannelongue, G.; Queiruga, D. Stakeholders and environmental management systems: a synergistic influence on environmental imbalance. Journal of Cleaner Production, 19, 1622-1630, 2011. doi: 10.1016/j. jclepro.2011.05.013

Heras-Saizarbitoria, I.; Landín, G. A.; Molina-Azorín, J. F. Do drivers matter for the benefits of ISO 14001? International Journal of Operations \& Production Management, 31(2), 192-216, 2011. doi: 10.1108/01443571111104764

ISO - International Organization for Standardization. The ISO Survey of ISO 9000 and ISO 14000 Certificates: 10th Cycle. Génova, 2000. Disponível em: <http://www.iso.org/ iso/survey10thcycle.pdf>. Acesso em: out. 2014.

ISO - International Organization for Standardization. ISO 14001: 2004. Genebra, 2004. Disponível em: <www. iso.org/iso/home/store/catalogue_tc/catalogue_detail. htm? csnumber $=31807>$. Acesso em: abr. 2015.

ISO - International Organization for Standardization. Environmental Management: The ISO 14000 Family of 
International Standards. Genebra, 2009. Disponível em: $<$ www.iso.org/iso/theiso14000family_2009.pdf>. Acesso em: jun. 2015.

ISO - International Organization for Standardization. ISO Survey. Genebra. 2015. Disponível em: <http:// www.iso.org/iso/home/standards/certification/iso-survey. $\mathrm{htm}$ ? certificate $=\mathrm{ISO} \% 209001 \&$ countrycode $=\mathrm{AF}>$. Acesso em: set. 2015 .

ISO - International Organization for Standardization. About ISO. Genebra. 2015a. Disponível em: <http://www.iso.org/ iso/home/about.htm>. Acesso em: jul. 2015.

ISO - International Organization for Standardization. Standards catalogue: ISO/TC 207/SC1 - Environmental management systems. Genebra, 2015b. Disponível em: <http:// www.iso.org/iso/home/store/catalogue_tc/catalogue_tc browse.htm?commid=54818>. Acesso em: jul. 2015.

ISO - International Organization for Standardization. The newly revised ISO 14001 is here. Genebra, 2015c. Disponível em: <http://www.iso.org/iso/news.htm?refid=Ref1999>. Acesso em: dez. 2015.

Jiang, R. J.; Bansal, P. Seeing the need for ISO 14001. Journal of Management Studies, 40(4), 1047-1067, 2003. doi: 10.1111/1467-6486.00370

Khan, Z. Cleaner production: an economical option for ISO certification in developing countries. Journal of Cleaner Production, 16(1), 22-27, 2008.

Kola-Lawal, C. et al. Factors in Organizational Environmental Management System Implementation-Developed vs. Developing Country Contexts. Journal of Sustainable Development of Energy, Water and Environment Systems, 2(4), 408-421, 2014. doi: 10.13044/j.sdewes.2014.02.0032

Kollman, K.; Prakash, A. EMS-based environmental regimes as club goods: examining variations in firmlevel adoption of ISO 14001 and EMAS in UK, US and Germany. Policy Sciences, 35(1), 43-67, 2002. doi: 10.1023/A:1016071810725

Lukács, E. The economic role of SMEs in world economy, especially in Europe. European Integration Studies, 1(4), 3-12, 2005. Disponível em: <www.uni-miskolc.hu/uni/ res/.../2005/.../lukacs.doc $>$.

Malik, K. et al. Human Development Report 2014. Sustaining Human Progress:
Reducing Vulnerabilities and Building Resilience. Nova York: United Nations Development Programme, 2014.

Marimon, F.; Casadesús, M.; Heras-Saizarbitoria, I. ISO 9000 and ISO 14000 standards: an international diffusion model. International Journal of Operations \& Production Management, 26(2), 141-165, 2006. doi: 10.1108/01443570610641648

Marimon, F.; Casadesús, M.; Heras-Saizarbitoria, I. Certification intensity level of the leading nations in ISO 9000 and ISO 14000 standards. International Journal of Quality \& Reliability Management, 27(9), 1002-1020, 2010. doi: $10.1108 / 02656711011084800$

Massoud, M. A. et al. Drivers, barriers and incentives to implementing environmental management systems in the food industry: A case of Lebanon. Journal of Cleaner Production, 18(3), 200-209, 2010a. doi: 10.1016/j.jclepro.2009.09.022

Massoud, M. A. et al. Environmental management system (ISO 14001) certification in developing countries: challenges and implementation strategies. Environmental Science \& Technology, 44(6), 1884-1887, 2010b. doi: 10.1021/es902714u

Mauri, C. Environmental law enforcement and compliance in Central America. In: Annals of Sixth International Conference on Environmental Compliance and Enforcement. San José, 2002. p. 1-21.

Montiel, I.; Husted, B.W. The adoption of voluntary environmental management programs in Mexico: First movers as institutional entrepreneurs. Journal of Business Ethics, 88(2), 349-363, 2009. doi: 10.1007/s10551-009-0282-y

Neumayer, E.; Perkins, R. What explains the uneven takeup of ISO 14001 at the global level? A panel data analysis. Environment and Planning A, 36, 823-839, 2004. doi: 10.1068/a36144

Newbold, J. Chile's environmental momentum: ISO 14001 and the large-scale mining industry-case studies from the state and private sector. Journal of Cleaner Production, 14(3), 248-261, 2006. doi:10.1016/j.jclepro.2004.05.010

Nguyen, Q. A.; Hens, L. Environmental performance of the cement industry in Vietnam: the influence of ISO 14001 certification. Journal of Cleaner Production, 96(1), 362378, 2013. doi: 10.1016/j.jclepro.2013.09.032

Nishitani, K. Demand for ISO 14001 adoption in the global supply chain: An empirical analysis focusing on 
environmentally conscious markets. Resource and Energy Economics, 32(3), 395-407, 2010. doi: 10.1016/j.reseneeco.2009.11.002

Poksinska, B.; Dahlgaard, J. J.; Eklund, J. A. E. Implementing ISO 14000 in Sweden: motives, benefits and comparisons with ISO 9000. International Journal of Quality \& Reliability Management, 20(5), 585-606, 2003. doi: 10.1108/02656710310476543

Pombo, F. R.; Magrini, A. Panorama de aplicação da norma ISO 14001 no Brasil. Gestão \& Produção, 15(1), 1-10, 2008. doi: 10.1590/S0104-530X2008000100002

Potoski, M.; Prakash, A. Green clubs and voluntary governance: ISO 14001 and firms' regulatory compliance. American Journal of Political Science, 49(2), 235-248, 2005. doi: 10.1111/j.0092-5853.2005.00120.x

The World Bank. World Development Indicators. Washington, D.C.: The World Bank (producer and distributor). Disponível em: <http://data.worldbank.org/data-catalog/ world-development-indicators $>$. Acesso em: jun. 2015.

To, W. M.; Lee, P. K. C. Diffusion of ISO 14001 environmental management system: global, regional and countrylevel analyses. Journal of Cleaner Production, 66, 489-498, 2014. doi: 10.1016/j.jclepro.2013.11.076
Vastag, G. Revisiting ISO 14000 diffusion: A new "look" at the drivers of certification. Production and Operations Management, 13(3), 260-267, 2004. doi: 10.1111/j.19375956.2004.tb00510.x

Viadiu, F. M.; Fa, M. C.; Heras-Saizarbitoria, I. H. ISO 9000 and ISO 14000 standards: an international diffusion model. International Journal of Operations \& Production Management, 26(2), 141-165, 2006. doi: 10.1108/01443570610641648

Woodside, G.; Aurrichio, P.; Yturri, J. ISO 14001 Implementation Manual. New York: McGraw-Hill, 1998.

World Trade Organization. World Trade Report 2014. Trade and development: recent trends and the role of the WTO. World Trade Organization, 2014.

Wu, S-Y.; Chu, P-Y.; Liu, T-Y. Determinants of a firm's ISO 14001 certification: an empirical study of Taiwan. Pacific Economic Review, 12(4), 467-487, 2007. doi: 10.1111/j.1468-0106.2007.00365.x

Zeng, S. X. et al. Towards implementation of ISO 14001 environmental management systems in selected industries in China. Journal of Cleaner Production, 13(7), 645-656, 2005. doi: 10.1016/j.jclepro.2003.12.009 